The Egyptian Journal of Hospital Medicine (January 2021) Vol. 82 (3), Page 461-470

\title{
Environmental Risk Factors Associated with Children Autism Spectrum Disorders in, Menoufia Governorate Nagwa Nashat Hegazy ${ }^{1}$, Seham Mohammed Ragab ${ }^{2}$, Walaa Zaki El Hofey ${ }^{* 1}$ \\ Departments ${ }^{1}$ Family Medicine and ${ }^{2}$ Pediatric, Faculty of Medicine, Menoufia University, Menoufia, Egypt \\ *Corresponding author: Walaa Zaki El Hofey, Mobile: (+20) 01000502830, E-Mail: walaazaki2211@ @otmail.com
}

\begin{abstract}
Background: Although no official statistics are available, some medical studies suggest that there are over 800,000 children with autism in Egypt. While, there is no cure for autism spectrum disorder, intensive, early treatment can make a big difference in the lives of many children.

Objective: To explore environmental risk factors for autism spectrum disorders by using participants identified through broad ascertainment and reliable classification in Shebin El Kom city.

Subjects and Methods: Our study was conducted at Psychiatric and Neurology Clinic, Pediatric Department, Menoufia University Hospital and centers for children with special needs in Shebin El Kom City. A case control study enrolled 80 children, 40 of them diagnosed with autistic spectrum disorders by DSM-V-TR criteria with no another medical disease and another 40 apparently healthy children, from their brothers as a control group. All children attended to Psychiatric and Neurology Clinic, Pediatric Department, Menoufia University Hospital in Shebin El Kom City, Menoufia Governorate, Egypt during the period from April 2017 to January 2019.

Results: There were significant differences between cases and control groups regarding gender, history of hypertension, diabetes mellitus, folic-iron, fever, bleeding, induced labor and precipitous labor, dental fillings, silver/mercury fillings in pregnancy and taking antibiotics during pregnancy or breast fed, born healthy $>4.5 \mathrm{~kg}$, neonatal unit, breast fed, Jaundice, delayed sitting, delayed crawling, delayed walking and delayed speaking.

Conclusions: From the current study, it was concluded that boys, high and moderate socioeconomic states and positive family history represented conditions associated with an increased risk for autism and ASDs in Egyptian population.
\end{abstract}

Keywords: Autism, Fetal Factors, Maternal Factors, Risk Factors.

\section{INTRODUCTION}

Autism spectrum disorder (ASD) is a serious neurodevelopmental disorder that impairs child's ability to communicate and interact with others. It also includes restricted repetitive behaviors, interests and activities. These issues cause significant impairment in social, occupational and other areas of functioning ${ }^{(\mathbf{1})}$. The term (spectrum) in autism spectrum disorder refers to the wide range of symptoms and severity. The number of children diagnosed with autism spectrum disorder is rising. It is not clear whether this is due to better detection and reporting or areal increase in the number of cases or both ${ }^{(2)}$. Autism spectrum disorder affects children of all races and nationalities, but certain factors increase a child's risk. Environmental risk factors for autism may be broadly defined as any nongenetic contributor to the risk of autism and include reproductive-, dietary-, chemical-, and demographicrelated exposures. They include the child sex where boys are about four times more likely to develop ASD than girls do. Family history: families have one child with ASD have increased risk of having another child with the disorder. Extremely preterm babies. Parent's age: There may also be a connection between children boom to older parents and ASD. Other disorders: Fragile $\mathrm{X}$ syndrome, Tuberous sclerosis and Rett syndrome ${ }^{(3)}$. Although no official statistics are available, some medical studies suggest that there are over 800,000 children with autism in Egypt. While, there is no cure for autism spectrum disorder, intensive, early treatment can make a big difference in the lives of many children ${ }^{(4)}$. Early identification of autism spectrum disorder (ASD) is essential to ensure that children can access specialized evidence-based interventions that can help to optimize long-term outcomes. Early identification can be done through the Antenatal Primary health care settings through screening. It also helps shorten the stressful "diagnostic odyssey" that many families experience before diagnosis

Therefore, the aim of this study was to determine the possible risk factors for autistic spectrum disorders and assessment of the quality of life for their parents.

\section{SUBJECTS AND METHODS}

Our study was conducted at Psychiatric and Neurology Clinic, Pediatric Department, Menoufia University Hospital and centers for children with special needs in Shebin El Kom city.

A case control study was conducted on 80 children, 40 of them diagnosed with autistic spectrum disorders by DSM-V-TR criteria with no another medical disease 
and another 40 apparently healthy children, from their brothers as a control group. The study was conducted through the period from April 2017 to January 2019.

Cases group: included 40 children ( 31 boys and 9 girls), with ages ranging from 2 to 11 years old with mean of $6.5 \pm 2.5$ years and diagnosed with ASDs on the basis of the DSM- V-TR criteria.

Control group: enrolled 40 apparently healthy children (15 boys and 25 girls), with ages ranging from 2 to 11 years old, from their brothers. All controls were also clinically examined by the pediatricians to exclude the possibility to have any subclinical autistic features.

\section{Ethical consideration:}

The study was approved by the Ethical Committee of Menoufia Faculty of Medicine and informed consents were obtained from all subjects' guardian before the study was commenced.

\section{Selection Criteria for the Patients:}

The subjects included in this study were selected according to inclusion and exclusion criteria.

Inclusion criteria: Children with ASDs based on the DSM- V-TR criteria and aged from 2 to 11 years old. Exclusion criteria: Chronic disease (renal, cancer.... etc.) and Refusal to participate.

\section{All cases were subjected to the following:}

Detailed history taking with special emphasis on; onset course and duration of the disease, age and sex of the patient consanguinity. Antenatal or maternal history: age at patient's birth, history of chronic illness as hypertension, diabetes mellitus (DM) obesity, depression, epilepsy and PCO.

\section{During pregnancy:}

Medications (e.g. antiepileptic drugs) fever, bleeding, smoking. Natal and postnatal history including, gestational age, complication during labor or delivery, history of prematurity, gestational age at birth, birth weight, perinatal problems and postnatal course especially occurrence of neonatal hypoxia and jaundice.

\section{Breast-feeding}

Developmental history (both mental and motor): age of sitting up without support, walking unassisted, first spoken word and history of vaccination. Past history including major childhood illnesses, any previous therapies used to treat the child's condition. Family history for any similar conditions, any genetic diseases and other psychological or mental disorders in the family. History of exposure to heavy metals, dental fillings, silver/mercury fillings in pregnancy, near factory, industry, highway residence, anti-D inject, taking antibiotics during pregnancy or breast-fed.

\section{Assessment of the quality of life:}

Overall quality of Life and general health, physical health domain, psychological domain, social relationships, spiritual life and environmental domain. Data were collected through complete history taking, previously prepared questionnaire for assessment of risk factors of autism spectrum disorders, scaling the socioeconomic level of their families and assessment of the quality of life by WHOQOL-BREF Questionnaire.

\section{Statistical Analysis}

Results were tabulated and statistically analyzed using a personal computer using MICROSOFT EXCEL 2016 and SPSS v. 21 (SPSS Inc., Chicago, IL, USA). Statistical analysis was done using: Descriptive: e.g. percentage (\%), mean and standard deviation. Analytical: that included chi-Squared $\left(\chi^{2}\right)$, and estimated prevalence odds ratios for fetal and maternal factors in relation to autism spectrum disorders by calculating odds ratios (ORs) and 95\% confidence intervals (CIs) using logistic regression. A value of $\mathrm{P}$ equal or less than 0.05 was considered statistically significant.

\section{RESULTS}

Results showed that there were highly significant differences between cases and control groups regarding gender, history of hypertension, and diabetes mellitus. On the other hand, there was no statistically significant differences between the studied groups regarding age, relatives, obesity, depression, epilepsy and PCO (Table 1). 
Table (1): Prenatal risk factors associated with Autism among the studied group

\begin{tabular}{|c|c|c|c|c|c|c|c|}
\hline \multirow[t]{2}{*}{ Parameter } & \multicolumn{2}{|c|}{ Cases } & \multicolumn{2}{|c|}{ Control } & \multirow{2}{*}{$\begin{array}{l}\text { Chi } \\
\text { square }\end{array}$} & \multirow[t]{2}{*}{ p-value } & \multirow{2}{*}{$\begin{array}{l}\text { Odd's ratio } \\
\text { (CI) }\end{array}$} \\
\hline & $\begin{array}{l}\text { No } \\
40\end{array}$ & $\begin{array}{c}\% \\
100\end{array}$ & $\begin{array}{l}\text { No } \\
40\end{array}$ & $\begin{array}{c}\% \% \\
100\end{array}$ & & & \\
\hline Age (mean \pm SD & \multicolumn{2}{|c|}{$6.55 \pm 2.552$} & \multicolumn{2}{|c|}{$7.28 \pm 3.909$} & 2.029 & $0.330^{\mathrm{ns}}$ & --- \\
\hline $\begin{array}{l}\text { Gender } \\
\text { Male } \\
\text { Female * }\end{array}$ & $\begin{array}{c}31 \\
9\end{array}$ & $\begin{array}{l}77.5 \\
22.5\end{array}$ & $\begin{array}{l}15 \\
25\end{array}$ & $\begin{array}{l}37.5 \\
62.5\end{array}$ & 13.095 & $<0.001$ & $\begin{array}{c}5.741^{* *} \\
(2.15-15.297)\end{array}$ \\
\hline $\begin{array}{l}\text { Relatives } \\
\text { No } \\
\text { Yes }\end{array}$ & $\begin{array}{c}33 \\
7\end{array}$ & $\begin{array}{l}82.5 \\
17.5\end{array}$ & $\begin{array}{c}33 \\
7\end{array}$ & $\begin{array}{l}82.5 \\
17.5\end{array}$ & 0.000 & $0.615^{\mathrm{ns}}$ & ---- \\
\hline $\begin{array}{l}\text { Hypertension } \\
\text { No * } \\
\text { Yes }\end{array}$ & $\begin{array}{c}32 \\
8\end{array}$ & $\begin{array}{l}80.0 \\
20.0\end{array}$ & $\begin{array}{c}40 \\
0\end{array}$ & $\begin{array}{c}100.0 \\
0.0\end{array}$ & 8.889 & 0.003 & $\begin{array}{c}21.185 * * \\
(1.178-380.92)\end{array}$ \\
\hline $\begin{array}{l}\text { Diabetes mellitus } \\
\text { No* } \\
\text { Yes }\end{array}$ & $\begin{array}{l}28 \\
12\end{array}$ & $\begin{array}{l}70.0 \\
30.0\end{array}$ & $\begin{array}{c}40 \\
0\end{array}$ & $\begin{array}{c}100.0 \\
0.0\end{array}$ & 14.118 & $<0.001$ & $\begin{array}{c}35.526 * * \\
(2.02-624.75)\end{array}$ \\
\hline $\begin{array}{l}\text { Obesity } \\
\text { No } \\
\text { Yes }\end{array}$ & $\begin{array}{c}35 \\
5\end{array}$ & $\begin{array}{l}87.5 \\
12.5\end{array}$ & $\begin{array}{c}36 \\
4\end{array}$ & $\begin{array}{l}90.0 \\
10.0\end{array}$ & 0.125 & $0.500^{\mathrm{ns}}$ & --- \\
\hline $\begin{array}{l}\text { Depression } \\
\text { No } \\
\text { Yes }\end{array}$ & $\begin{array}{c}38 \\
2\end{array}$ & $\begin{array}{c}95.0 \\
5.0\end{array}$ & $\begin{array}{c}40 \\
0\end{array}$ & $\begin{array}{c}100.0 \\
0.0\end{array}$ & 2.051 & $0.247^{\mathrm{ns}}$ & -- \\
\hline Epilepsy & & & & cases & & & -- \\
\hline $\begin{array}{l}\mathrm{PCO} \\
\text { No } \\
\text { Yes }\end{array}$ & $\begin{array}{c}35 \\
5\end{array}$ & $\begin{array}{l}87.5 \\
12.5\end{array}$ & $\begin{array}{c}38 \\
2\end{array}$ & $\begin{array}{c}95.0 \\
5.0\end{array}$ & 1.409 & $0.216^{\mathrm{ns}}$ & -- \\
\hline
\end{tabular}

SD: stander deviation t: student t test $\mathrm{X}^{2}$ : chi square OR: Odd's ratio CI confidence interval ${ }^{\text {ns }}$ means not significant *reference group.

The current study showed that there was a significant difference between cases and control groups regarding folic-iron, fever, bleeding, induced labor and precipitous labor. On the other hand, there was no statistically significant differences between the studied groups regarding drugs taken, smoking, vaginal labor and prolonged labor. Results of the present study concluded that, there were statistically significant differences between cases and control groups regarding dental fillings, silver/mercury fillings in pregnancy and taking antibiotics during pregnancy or breast-fed. While, there was no statistically significant differences between the studied groups regarding near factory, industry, highway residence and Rhogam injections just after childbirth (Table 2). 
Table (2): Perinatal and postnatal factors associated with Autism among the studied group

\begin{tabular}{|c|c|c|c|c|c|c|c|}
\hline \multirow[t]{2}{*}{ Parameter } & \multicolumn{2}{|c|}{ Cases } & \multicolumn{2}{|c|}{ Control } & \multirow{2}{*}{$\begin{array}{c}\text { Chi } \\
\text { square }\end{array}$} & \multirow[t]{2}{*}{ p-value } & \multirow{2}{*}{$\begin{array}{l}\text { Odd's ratio } \\
\text { (CI) }\end{array}$} \\
\hline & $\begin{array}{l}\text { No } \\
40\end{array}$ & $\begin{array}{c}\% \\
100\end{array}$ & $\begin{array}{c}\text { No } \\
40\end{array}$ & $\begin{array}{c}\% \\
100\end{array}$ & & & \\
\hline $\begin{array}{l}\text { Folic-iron } \\
\text { No } \\
\text { Yes* }\end{array}$ & $\begin{array}{c}8 \\
32\end{array}$ & $\begin{array}{l}80.0 \\
45.7\end{array}$ & $\begin{array}{c}2 \\
38\end{array}$ & $\begin{array}{l}20.0 \\
54.3\end{array}$ & 4.114 & 0.044 & $\begin{array}{c}0.211 \\
(0.042-1.06)\end{array}$ \\
\hline $\begin{array}{l}\text { Drugs } \\
\text { No } \\
\text { Yes }\end{array}$ & $\begin{array}{c}39 \\
1\end{array}$ & $\begin{array}{c}49.4 \\
100.0\end{array}$ & $\begin{array}{c}40 \\
0\end{array}$ & $\begin{array}{c}50.6 \\
0.0\end{array}$ & 1.013 & $0.500^{\mathrm{ns}}$ & --- \\
\hline $\begin{array}{l}\text { Fever } \\
\text { No* } \\
\text { Yes }\end{array}$ & $\begin{array}{c}32 \\
8 \\
\end{array}$ & $\begin{array}{c}44.4 \\
100.0\end{array}$ & $\begin{array}{c}40 \\
0 \\
\end{array}$ & $\begin{array}{c}55.6 \\
0.0\end{array}$ & 8.889 & 0.003 & $\begin{array}{c}21.185^{* *} \\
(1.18-380.9)\end{array}$ \\
\hline $\begin{array}{l}\text { Bleeding } \\
\text { No* } \\
\text { Yes }\end{array}$ & $\begin{array}{c}31 \\
9\end{array}$ & $\begin{array}{l}44.3 \\
90.0\end{array}$ & $\begin{array}{c}39 \\
1\end{array}$ & $\begin{array}{l}55.7 \\
10.0\end{array}$ & 7.314 & 0.007 & $\begin{array}{c}11.323 * * \\
(1.36-94.25)\end{array}$ \\
\hline $\begin{array}{l}\text { Smoking } \\
\text { No } \\
\text { Yes }\end{array}$ & $\begin{array}{c}34 \\
6 \\
\end{array}$ & $\begin{array}{l}47.9 \\
66.7 \\
\end{array}$ & $\begin{array}{c}37 \\
3 \\
\end{array}$ & $\begin{array}{l}52.1 \\
33.3 \\
\end{array}$ & 1.127 & $0.241^{\mathrm{ns}}$ & -- \\
\hline $\begin{array}{l}\text { Vaginal labor } \\
\text { No } \\
\text { Yes }\end{array}$ & $\begin{array}{l}22 \\
18\end{array}$ & $\begin{array}{l}55.0 \\
45.0\end{array}$ & $\begin{array}{l}18 \\
22\end{array}$ & $\begin{array}{l}45.0 \\
55.0\end{array}$ & 0.800 & $0.251^{\mathrm{ns}}$ & --- \\
\hline $\begin{array}{l}\text { Induced labor } \\
\text { No* } \\
\text { Yes }\end{array}$ & $\begin{array}{c}32 \\
8 \\
\end{array}$ & $\begin{array}{c}44.4 \\
100.0 \\
\end{array}$ & $\begin{array}{c}40 \\
0\end{array}$ & $\begin{array}{c}55.6 \\
0.0\end{array}$ & 8.889 & 0.003 & $\begin{array}{c}15.26 \\
(0.83-280.7)\end{array}$ \\
\hline $\begin{array}{l}\text { Precipitous labor } \\
\text { No* } \\
\text { Yes }\end{array}$ & $\begin{array}{c}35 \\
5\end{array}$ & $\begin{array}{l}46.7 \\
100.0\end{array}$ & $\begin{array}{c}40 \\
0\end{array}$ & $\begin{array}{c}53.3 \\
0.0\end{array}$ & 5.333 & 0.027 & $\begin{array}{c}12.55 \\
(0.67-235.01)\end{array}$ \\
\hline $\begin{array}{l}\text { Prolonged labor } \\
\text { No } \\
\text { Yes }\end{array}$ & $\begin{array}{c}36 \\
4 \\
\end{array}$ & $\begin{array}{c}47.4 \\
100.0 \\
\end{array}$ & $\begin{array}{c}40 \\
0 \\
\end{array}$ & $\begin{array}{c}52.6 \\
0.0 \\
\end{array}$ & 4.211 & $0.058^{\mathrm{ns}}$ & ---- \\
\hline $\begin{array}{l}\text { Dental fillings } \\
\text { No* } \\
\text { Yes }\end{array}$ & $\begin{array}{c}33 \\
7 \\
\end{array}$ & $\begin{array}{l}82.5 \\
17.5 \\
\end{array}$ & $\begin{array}{c}39 . \\
1 \\
\end{array}$ & $\begin{array}{c}97.5 \\
2.5 \\
\end{array}$ & 5.000 & 0.028 & $\begin{array}{c}8.273 \\
(0.967-70.736) \\
\end{array}$ \\
\hline $\begin{array}{l}\text { Silver/mercury fillings in pregnancy } \\
\text { No* } \\
\text { Yes }\end{array}$ & $\begin{array}{l}27 \\
13\end{array}$ & $\begin{array}{l}67.5 \\
32.5\end{array}$ & $\begin{array}{c}38 \\
2\end{array}$ & $\begin{array}{c}95.0 \\
5.0\end{array}$ & 9.928 & 0.002 & $\begin{array}{c}9.148 * * \\
(1.906-43.988)\end{array}$ \\
\hline $\begin{array}{l}\text { Near a factory, industry, highway } \\
\text { residence } \\
\text { No } \\
\text { Yes }\end{array}$ & $\begin{array}{c}32 \\
8\end{array}$ & $\begin{array}{l}87.5 \\
12.5\end{array}$ & $\begin{array}{c}35 \\
5\end{array}$ & $\begin{array}{l}80.0 \\
20.0\end{array}$ & 0.827 & $0.273^{\mathrm{ns}}$ & ---- \\
\hline $\begin{array}{l}\text { Rhogam injections just after childbirth } \\
\text { No } \\
\text { Yes }\end{array}$ & $\begin{array}{c}35 \\
5 \\
\end{array}$ & $\begin{array}{l}87.5 \\
12.5 \\
\end{array}$ & $\begin{array}{c}37 \\
3 \\
\end{array}$ & $\begin{array}{c}92.5 \\
7.5 \\
\end{array}$ & 0.556 & $0.356^{\mathrm{ns}}$ & ----- \\
\hline $\begin{array}{l}\text { Taking antibiotics during pregnancy or } \\
\text { breast fed } \\
\text { No* } \\
\text { Yes }\end{array}$ & $\begin{array}{l}27 \\
13 \\
\end{array}$ & $\begin{array}{l}67.5 \\
32.5 \\
\end{array}$ & $\begin{array}{c}40 \\
0 \\
\end{array}$ & $\begin{array}{c}100.0 \\
0.0 \\
\end{array}$ & 15.522 & $<0.001$ & $\begin{array}{c}39.7636^{* *} \\
(2.268-97.094)\end{array}$ \\
\hline
\end{tabular}

${ }^{n s}$ means not significant CI: Confidence interval *reference group

The current study revealed that, there were statistically significant differences between cases and control groups regarding born healthy (> $4.5 \mathrm{~kg}$ ), neonatal unit, breast-fed, jaundice, delayed sitting, delayed crawling, delayed 
walking and delayed speaking. While, there was no statistically significant differences between the studied groups regarding full term baby and post vaccination (Table 3 ).

Table (3): Fetal factors associated with Autism among the studied group

\begin{tabular}{|c|c|c|c|c|c|c|c|}
\hline \multirow[t]{2}{*}{ Parameter } & \multicolumn{2}{|c|}{ Cases } & \multicolumn{2}{|c|}{ Control } & \multirow[t]{2}{*}{ Chi square } & \multirow[t]{2}{*}{ p-value } & \multirow{2}{*}{$\begin{array}{l}\text { Odd's ratio } \\
\text { (CI) }\end{array}$} \\
\hline & $\begin{array}{l}\text { No } \\
40 \\
\end{array}$ & $\begin{array}{c}\% \\
100 \\
\end{array}$ & $\begin{array}{l}\text { No } \\
40 \\
\end{array}$ & $\begin{array}{c}\% \\
100 \\
\end{array}$ & & & \\
\hline $\begin{array}{l}\text { Born healthy } \\
\text { No } \\
\text { Yes* }\end{array}$ & $\begin{array}{c}9 \\
31\end{array}$ & $\begin{array}{l}22.5 \\
77.5\end{array}$ & $\begin{array}{c}0 \\
40\end{array}$ & $\begin{array}{c}0.0 \\
100.0\end{array}$ & 10.141 & 0.001 & $\begin{array}{c}0.0409 * * \\
(0.002-0.7305)\end{array}$ \\
\hline $\begin{array}{l}\text { Full term baby } \\
\text { No } \\
\text { Yes }\end{array}$ & $\begin{array}{c}4 \\
36 \\
\end{array}$ & $\begin{array}{l}10.0 \\
90.0\end{array}$ & $\begin{array}{c}0 \\
40 \\
\end{array}$ & $\begin{array}{c}0.0 \\
100.0 \\
\end{array}$ & 4.211 & $0.058^{\mathrm{ns}}$ & ---- \\
\hline $\begin{array}{l}>4.5 \mathrm{k} . \mathrm{g} \\
\text { No* } \\
\text { Yes }\end{array}$ & $\begin{array}{l}29 \\
11\end{array}$ & $\begin{array}{l}72.5 \\
27.5\end{array}$ & $\begin{array}{c}40 \\
0\end{array}$ & $\begin{array}{c}100.0 \\
0.0\end{array}$ & 12.752 & $<0.001$ & $\begin{array}{c}31.576^{* *} \\
(1.788-557.43)\end{array}$ \\
\hline $\begin{array}{l}\text { Neonatal unit } \\
\text { No* } \\
\text { Yes } \\
\end{array}$ & $\begin{array}{l}26 \\
14 \\
\end{array}$ & $\begin{array}{l}65.0 \\
35.0 \\
\end{array}$ & $\begin{array}{c}40 \\
0 \\
\end{array}$ & $\begin{array}{c}100.0 \\
0.0\end{array}$ & 16.97 & $<0.001$ & $\begin{array}{c}44.321^{* *} \\
(2.535-775.039) \\
\end{array}$ \\
\hline $\begin{array}{l}\text { Breast fed } \\
\text { No } \\
\text { Yes* }\end{array}$ & $\begin{array}{l}15 \\
25\end{array}$ & $\begin{array}{l}37.5 \\
62.5\end{array}$ & $\begin{array}{c}6 \\
34\end{array}$ & $\begin{array}{l}15.0 \\
85.0\end{array}$ & 5.23 & 0.02 & $\begin{array}{c}9.444 * * \\
(3.212-27.767)\end{array}$ \\
\hline $\begin{array}{l}\text { Jaundice } \\
\text { No* } \\
\text { Yes } \\
\end{array}$ & $\begin{array}{l}21 \\
19 \\
\end{array}$ & $\begin{array}{l}52.5 \\
47.5 \\
\end{array}$ & $\begin{array}{c}37 \\
3 \\
\end{array}$ & $\begin{array}{c}92.5 \\
7.5 \\
\end{array}$ & 16.05 & $<0.001$ & $\begin{array}{c}11.158 * * \\
(2.95-42.201) \\
\end{array}$ \\
\hline $\begin{array}{l}\text { Delayed Sitting } \\
\text { No* } \\
\text { Yes }\end{array}$ & $\begin{array}{c}33 \\
7 \\
\end{array}$ & $\begin{array}{l}82.5 \\
17.5 \\
\end{array}$ & $\begin{array}{c}40 \\
0 \\
\end{array}$ & $\begin{array}{c}100.0 \\
0.0\end{array}$ & 7.671 & 0.006 & $\begin{array}{c}18.134 \\
(0.998-329.288) \\
\end{array}$ \\
\hline $\begin{array}{l}\text { Delayed Crawling } \\
\text { No* } \\
\text { Yes } \\
\end{array}$ & $\begin{array}{c}33 \\
7 \\
\end{array}$ & $\begin{array}{l}82.5 \\
17.5 \\
\end{array}$ & $\begin{array}{c}40 \\
0 \\
\end{array}$ & $\begin{array}{c}100.0 \\
0.0 \\
\end{array}$ & 7.671 & 0.006 & $\begin{array}{c}18.134 \\
(0.998-329.288) \\
\end{array}$ \\
\hline $\begin{array}{l}\text { Delayed walking } \\
\text { No* } \\
\text { Yes }\end{array}$ & $\begin{array}{c}32 \\
8\end{array}$ & $\begin{array}{l}80.0 \\
20.0\end{array}$ & $\begin{array}{c}40 \\
0\end{array}$ & $\begin{array}{c}100.0 \\
0.0\end{array}$ & 8.889 & 0.003 & $\begin{array}{c}21.185^{* *} \\
(1.178-380.925)\end{array}$ \\
\hline $\begin{array}{l}\text { Delayed speaking } \\
\text { No* } \\
\text { Yes } \\
\end{array}$ & $\begin{array}{c}1 \\
39 \\
\end{array}$ & $\begin{array}{c}2.5 \\
97.5 \\
\end{array}$ & $\begin{array}{c}40 \\
0 \\
\end{array}$ & $\begin{array}{c}100.0 \\
0.0 \\
\end{array}$ & 76.098 & $<0.001$ & $\begin{array}{c}2133^{* *} \\
(84.33-53950.5) \\
\end{array}$ \\
\hline $\begin{array}{l}\text { Post vaccination } \\
\text { No } \\
\text { Yes } \\
\end{array}$ & $\begin{array}{c}35 \\
5\end{array}$ & $\begin{array}{l}87.5 \\
12.5\end{array}$ & $\begin{array}{c}39 \\
1 \\
\end{array}$ & $\begin{array}{c}97.5 \\
2.5\end{array}$ & 2.883 & $0.100^{\mathrm{ns}}$ & -- \\
\hline
\end{tabular}

${ }^{\mathrm{ns}}$ means not significant CI confidence interval *reference group

In the current study, fetal factors e.g. gender (OR, 11.11; 95\% CI, 1.419-86.96) and (OR, 10.62; 95\% CI, 1.16496.898) breast feeding and maternal factors e.g. bleeding, (OR, 34.377; 95\% CI, 1.835-644.007) and silver/mercury fillings in pregnancy (OR, 26.267; 95\% CI, 1.147-601.695) were significantly associated with risk of autism in the full study $(\mathrm{p}=0.022,0.036)$ and $(\mathrm{p}=0.018,0.041)$ respectively. While, other fetal and maternal factors were not associated with ASD risk $(\mathrm{p}>0.05)$ (Table 4$)$. 
Table (4): Fetal and maternal factors in relation to risk of ASD

\begin{tabular}{|c|c|c|c|c|}
\hline Items & B & $P$ value & OR & CI \\
\hline $\begin{array}{l}\text { Gender } \\
\text { Male } \\
\text { Female* }\end{array}$ & 2.345 & 0.022 & 11.11 & $1.419-86.96$ \\
\hline $\begin{array}{l}\text { Hypertension } \\
\text { No* } \\
\text { Yes } \\
\end{array}$ & 0.097 & $1.000^{\mathrm{ns}}$ & 1.220 & ---- \\
\hline $\begin{array}{l}\text { Diabetes mellitus } \\
\text { No* } \\
\text { Yes }\end{array}$ & 20.608 & $0.999^{\mathrm{ns}}$ & 8.178 & --- \\
\hline $\begin{array}{l}\text { Fever } \\
\text { No* } \\
\text { Yes }\end{array}$ & 16.49 & $0.999^{\mathrm{ns}}$ & 1.155 & ---- \\
\hline $\begin{array}{l}\text { Bleeding } \\
\text { No* } \\
\text { Yes } \\
\end{array}$ & 3.392 & 0.018 & 34.377 & $1.835-644.007$ \\
\hline $\begin{array}{l}\text { Silver/mercury fillings in pregnancy } \\
\text { No* } \\
\text { Yes }\end{array}$ & 3.041 & 0.041 & 26.267 & $1.147-601.695$ \\
\hline $\begin{array}{l}\text { Taking antibiotics during pregnancy } \\
\text { or breast fed } \\
\text { No } * \\
\text { Yes }\end{array}$ & 20.303 & $0.998^{\mathrm{ns}}$ & 8.745 & --- \\
\hline $\begin{array}{l}\text { Born healthy } \\
\text { No } \\
\text { Yes* }\end{array}$ & 19.829 & $0.998^{\mathrm{ns}}$ & 4.032 & --- \\
\hline $\begin{array}{l}>4.5 \mathrm{k.g} \\
\text { No* } \\
\text { Yes }\end{array}$ & 0.301 & $1.000^{\mathrm{ns}}$ & 1.207 & --- \\
\hline $\begin{array}{l}\text { Neonatal unit } \\
\text { No* } \\
\text { Yes }\end{array}$ & 18.82 & $0.998^{\mathrm{ns}}$ & 2.322 & --- \\
\hline $\begin{array}{l}\text { Breast fed } \\
\text { No } \\
\text { Yes* }\end{array}$ & 2.322 & 0.036 & 10.62 & $1.164-96.898$ \\
\hline $\begin{array}{l}\text { Delayed walking } \\
\text { No } * \\
\text { Yes }\end{array}$ & 20.049 & 0.999 ns & 7.771 & ---- \\
\hline
\end{tabular}

*reference group ${ }^{\mathrm{ns}}$ means not significant

\section{DISCUSSION}

In the current study, there were highly significant differences between cases and control groups regarding gender, history of hypertension, and diabetes mellitus. On the other hand, there was no statistically significant differences between the studied groups regarding age, relatives, obesity, depression, epilepsy and PCO. Similar findings were noted by Zhen-Huan et al. ${ }^{(5)}$ who evaluated the validity of existing QoL questionnaires for use with children with ASD aged 8-12 years. 200 autistic children (male: 118 , female: $82 ; 2 \sim 4$ years old: $80,5 \sim$ 7-year-old: $87,8 \sim 12$ years old: 33 ) and 120 normal children (control group) were brought into this study. Results found that there were statistically significant differences between the autistic children and normal children regarding gender $(\mathrm{p}=0.0102)$, age $(\mathrm{p}=0.0248)$, education level $(\mathrm{P}=0.0416)$, and family income/month $(\mathrm{P}=0.0434)$, suggesting comparable. Similarly, Guinchat et al. ${ }^{\left({ }^{6}\right)}$ in their review of 85 studies on the possible association between prenatal, perinatal and 
neonatal factors and ASD, they did not find a strong association of maternal diabetes and ASD. On the other hand, there were studies that could not demonstrate such associations. For example, Hultman et al. ${ }^{(7)}$ in their case control study on 408 Swedish children with ASD compared to 2040 matched controls. They found an association of ASD with a variety of pregnancyassociated factors but not with maternal diabetes. However, there are also studies demonstrated such associations. Maternal diabetes was among the leading factors associated with ASD, with an odds ratio of 2.07 (95\% CI 1.24-3.47) ${ }^{(8)}$.

Our findings indicated that there was a significant difference between cases and control groups regarding folic-iron, fever, bleeding, induced labor and precipitous labor. On the other hand, there was no statistically significant differences between the studied groups regarding drugs taken, smoking, vaginal labor and prolonged labor. This was comparable with folic acid deficiency, which was therefore also proposed as a possible risk factor for ASD. Schmidt et al. ${ }^{(9)}$ were the first to report that mothers of children with autism were less likely, than those of typically developing children, to report having prenatal vitamins, including folic acid, during the 3 months before pregnancy or the first month of pregnancy. Later, Schmidt et al. ${ }^{(9)}$ found that mothers of normal children had a significantly greater mean folic acid intake during the first month of pregnancy compared to mothers of children with ASD. The association between folic acid and reduced ASD risk was strongest for mothers and children with MTHFR 677C > $\mathrm{T}$ variant genotypes. Recently, DeVilish et al. (10) concluded that the data associating folate deficiency with a higher risk of autism is yet inconclusive.

Regarding, metabolic syndrome, bleeding, and mother infection during pregnancy. Maternal bleeding during pregnancy was associated with a significant $81 \%$ elevated risk of autism, and metabolic syndrome, including diabetes, hypertension, and obesity, paves the way for hypoxia in utero, which results in deficient brain development and induction of myelination changes, membrane adhesion, and deficiency in hippocampal neurons ${ }^{(6)}$.

Regarding smoking, other large studies Maimburg et al. ${ }^{(11)}$ and Burstyn et al. ${ }^{(12)}$ also did not find any association between tobacco exposure and ASD, which agrees with our results. Lee et al. ${ }^{(\mathbf{1 3})}$ found that maternal smoking during pregnancy is not associated with increased risk of ASD after adjustments for parental education, occupation, and income. Several additional studies and two recently published metaanalyses by Rosen et al. ${ }^{(14)}$ and Tang et al. ${ }^{(15)}$, also reported no significant association between maternal smoking, vaginal labor and prolonged labor during pregnancy and ASD. It can be summarized that maternal smoking does not increase the rate of ASD in the offspring.

Results of the present study concluded that there were statistically significant differences between cases and control groups regarding dental fillings, silver/mercury fillings in pregnancy and taking antibiotics during pregnancy or breast-fed. While, there was no statistically significant differences between the studied groups regarding near factory, industry, highway residence and Rhogam injections just after childbirth. These finding are consistent with study done by Geier $\boldsymbol{e t}$ al. (16) who found that a rise in autism severity was correlated with an elevation in the number of dental amalgams in the mother during pregnancy. Other study done by Gardener et al. ${ }^{(8)}$ reported that maternal prenatal medication use can be associated with a $46 \%$ increased risk of fetus autism. Researches about different kinds of drugs have revealed a significant (68\%) increased risk of autism in relation to prenatal psychiatric medication use ${ }^{(17)}$. The negative effect of prenatal medication use is caused by their placental crossing and disturbing fetal development, based on many studies. For example, use of antiepileptic drugs, as well as valproic acid leads to fetal valproate syndrome, increases oxidative stress and varied gene expression pattern, and subsequently results in developmental delays, deficient motor activities and social behaviors, and finally, postnatal growth alterations ${ }^{(18)}$. Also, Zablotsky et al. (19) showed that higher autism symptomatology and a greater number of co-occurring psychiatric disorders in the child were associated with an increased risk for current treatment of maternal depression and a lower maternal quality of life. While, in the study by Volk $\boldsymbol{e t}$ al. ${ }^{(20)}$ they found that maternal residence during the third trimester and at the time of delivery were more likely to be near a freeway $(\leq 309 \mathrm{~m})$ for the ASD children than for the controls. They also found that during gestation, children with ASD were more likely to live at residencies that had the highest quartile of exposure to traffic-related air pollution compared with control children. Regional exposure during pregnancy with nitrogen dioxide and particulate matter $<2.5$ and $10 \mu \mathrm{m}$ in diameter (PM2.5 and PM10) were also associated with autism.

The current study revealed that, there were statistically significant differences between cases and control groups regarding born healthy (> $4.5 \mathrm{~kg}$ ), neonatal unit, breast-fed, jaundice, delayed sitting, delayed crawling, delayed walking and delayed speaking. While, there was no statistically significant differences between the studied groups regarding fullterm baby and post-vaccination. Similar findings are noted by Karimi et al. ${ }^{(17)}$ who reported that low birth weight, jaundice, and postnatal infection are some of the 
most significant risk factors. A neonate with birth weight, which is the result of three potential factors minor than $2500 \mathrm{~g}$ considered as low birth weight and associated with a two-fold increase in the risk of autism $(\mathbf{8}, \mathbf{2 1}$. Postnatal jaundice is a result of high bilirubin production caused by increased breakdown of fetal erythrocytes and a low hepatic excretory capacity resulting from general immaturity of the liver and it can be associated with death during a sensitive period or susceptibility to mental disorders, especially a four-fold increase in autism if survive ${ }^{\text {(22) }}$.

In addition to prenatal maternal infection during pregnancy, postnatal infections such as meningitis, mumps, varicella, unknown fever, and ear infections on the first 30 days of life are correlated with high risk of autism ${ }^{(17)}$. Furthermore, Roumen et al. ${ }^{(23)}$ found that postnatal complications like birth asphyxia have an association with autism, which correlates with the findings of Finnegan and Arrington. On the other hand, Kheir et al. ${ }^{(24)}$ indicated that there was no significant difference between quality of life domains between the two groups of parents, but autistic children rated their health as poor and likely to get worse.

In the current study, fetal factors e.g. gender (OR, $11.11 ; 95 \% \mathrm{CI}, 1.419-86.96)$ and (OR, 10.62; 95\% CI, 1.164-96.898) breast feeding and maternal factors e.g. bleeding, (OR, 34.377; 95\% CI, 1.835-644.007) and silver/mercury fillings in pregnancy (OR, 26.267; 95\% CI, 1.147-601.695) were significantly associated with risk of autism in the full study $(\mathrm{p}=0.022,0.036)$ and $(\mathrm{p}=0.018,0.041)$ respectively. These results are consistent with the findings of Halladay $\boldsymbol{e t}$ al. ${ }^{(25)}$ who found that females with an ASD diagnosis and IQ scores within the average range showed increased functional social behavior compared to males with ASD. Females with ASD also show less repetitive behaviors compared to males. However, a stakeholder pointed out that the quality of these repetitive behaviors may be different. For example, a young woman with autism noted that carrying several well-worn books everywhere she goes, and constantly reading them to the detriment of all other social interactions, may be a repetitive behavior that goes undetected ${ }^{(26)}$. In addition, Werling and Geschwind ${ }^{(27)}$ demonstrated that ASD is consistently over-represented in males as compared to females. However, we currently do not understand the extent of this over-representation, or the degree to which this male bias in prevalence is related to intellectual functioning or ascertainment methods in addition to the influence of sex-differential genetic or hormonal factors. In contrast with the higher proportion of diagnosed autistic females than males with ID, many studies found no sex differences in overall composite ASD severity as measured on several standard assessment tools ${ }^{(28,29)}$. This suggests that among those who meet diagnostic criteria, females are not more severely affected. However, differences emerge when each core symptom domain of ASD is considered separately, and sex differences observed in cases tend to reflect sex differences observed in the typical population.

Regarding, breast feeding, Halladay et al. ${ }^{(25)}$ reported that in terms of autism risk, a number of research reports have considered breastfeeding versus formula. None directly tested whether breast milk from the breast or a bottle reduces or increases risk for autism spectrum disorder (ASD). However, most showed that children with ASD are more likely to have been either bottle fed from birth or weaned early than are comparison groups of children not affected by ASD. Also, Manohar et al. ${ }^{(30)}$ found that children who had received exclusive breastfeeding had decreased odds of being diagnosed with $\mathrm{ASD}(\mathrm{OR}=0.167,95 \% \mathrm{CI}=$ $0.025-0.65, \mathrm{P}=0.0162$ ), whereas those with early introduction of top feeds had increased odds $(\mathrm{OR}=6.0$, $95 \% \mathrm{CI}=1.33-55.19, \mathrm{P}=0.0162$ ).

Regarding, maternal factors, Previc et al. (31) reported that prenatal risk factors such as bleeding during pregnancy and natal risk factors such as fetal complications including fetal distress, umbilical-cord complications such as fetal nuchal cord and cesarean delivery (26\% increased risk of autism) are all involved in hypoxia and consequently increasing susceptibility to child autism ${ }^{(8)}$. In addition, Hvidtjørn et al. ${ }^{(32)}$ found that abnormal gestational age, preterm ( $<35$ weeks) and post term pregnancy ( $>42$ weeks), was associated with a significantly increased risk of autism. While, Kalkbrenner et al. ${ }^{(33)}$ examined prenatal exposure to 35 hazardous air pollutants in 383 children with ASD and 2829 control children with speech and language impairment. They found that prenatal exposure to hazardous air pollutants including methylene chloride (OR, 1.4; 95\% CI, 0.7-2.5), quinolone (OR, 1.4; 95\% CI, 1.0-2.2), and styrene (OR, 1.8; 95\% CI, 1.0-3.1) were associated with a higher risk for ASD.

\section{CONCLUSION}

From the current study, it was concluded that boys, high and moderate socioeconomic states and positive family history represented conditions associated with an increased risk for autism and ASDs in Egyptian population. There was a significant difference between the studied groups regarding folic-iron, fever, bleeding, induced labor and precipitous labor, dental fillings, silver/mercury fillings in pregnancy and taking antibiotics during pregnancy or breast fed, born healthy ( $>4.5 \mathrm{~kg}$ ), neonatal unit, breast fed, jaundice, delayed sitting, delayed crawling, delayed walking and delayed speaking. Also, fetal factors e.g. gender and breast feeding and maternal factors e.g. bleeding, and 
silver/mercury fillings in pregnancy were significantly associated with the risk of autism.

\section{REFERENCES}

1. Blanche E, Reinoso G, Chang $M$ et al. (2012): Proprioceptive processing difficulties among children with autism spectrum disorders and developmental disabilities. Am. J. Occup. Ther., 66: 621-624

2. Volkmar F, Siegel M, Woodbury-Smith M (2014): Practice parameter for the assessment and treatment of children and adolescents with autism spectrum disorder. JAm Acad Child Adolesc Psychiatry, 53: 237 257

3. Cheuk D, Wong $\mathrm{V}$, Chen $\mathrm{W}$ et al. (2011): Acupuncture for autism spectrum disorders (ASD): Cochrane Database of Systematic Reviews, 9: 142-148.

4. Weissman L, Bridgemohan $\mathrm{C}$, Augustyn $\mathrm{M}$ et al. (2014): Autism spectrum disorder in children and adolescents: Complementary and alternative therapies. https://www.mayoclinic.org/diseasesconditions/autism-spectrum-disorder/diagnosistreatment/drc-20352934

5. Zhen-Huan L, Qiao-Ling $X$, Zhang-Yong $X$ et al. (2016): Quality of Life of Children with ASD. Autism Open Access, 6:183-186.

6. Guinchat V, Thorsen P, Laurent $C$ et al. (2012): Preperi- and neonatal risk factors for autism. Acta Obstet Gynecol Scand., 91 (3): 287-300.

7. Hultman C, Sparén P, Cnattingius S (2002): Perinatal risk factors for infantile autism. Epidemiology, 13 (4): 417-23

8. Gardener H, Spiegelman D, Buka S (2009): Prenatal risk factors for autism: comprehensive meta-analysis: J Psychiatry, 195 (1): 7-14.

9. Schmidt R, Tancredi D, Ozonoff S et al. (2012): Maternal periconceptional folic acid intake and risk of autism spectrum disorders and developmental delay in the CHARGE (Childhood Autism Risks from Genetics and Environment) case-control study. Am J Clin Nutr., 96 (1): 80-89.

10. DeVilbiss E, Gardner R, Newschaffer C et al. (2015): Maternal folate status as a risk factor for autism spectrum disorders: a review of existing evidence. $\mathrm{Br} \mathrm{J}$ Nutr., 114 (5): 663-72.

11. Maimburg R, Bech B, Vaeth $M$ et al. (2010): Neonatal jaundice, autism, and other disorders of psychological development. Pediatrics, 126 (5): 872-8.

12. Burstyn I, Sithole F, waigenbaum $Z$ (2010): Autism spectrum disorders, maternal characteristics and obstetric complications among singletons born in Alberta, Canada. Chronic Dis Can., 30 (4): 125-34.

13. Lee B, Gardner R, Dal H et al. (2012): Brief report: maternal smoking during pregnancy and autism spectrum disorders. J Autism Dev Disord., 42 (9): 20005.

14. Rosen B, Lee B, Lee $\mathbf{N}$ et al. (2015): Maternal Smoking and Autism Spectrum Disorder: A Metaanalysis. Dev Discord., 45 (6): 1689-98.
15. Tang S, Wang Y, Gong $X$ et al. (2015): A MetaAnalysis of Maternal Smoking during Pregnancy and Autism Spectrum Disorder Risk in Offspring Int $\mathbf{J}$ Environ Res Public Health, 12 (9): 10418-31.

16. Geier D, Kern J, Geier M (2009): A prospective study of prenatal mercury exposure from maternal dental amalgams and autism severity. Acta Neurobiol Exp (Wars), 69 (2): 189- 97.

17. Karimi P, Kamali E, Mousavi S et al. (2017): Environmental factors influencing the risk of autism. Journal of research in medical sciences: the official journal of Isfahan University of Medical Sciences, 22: 27-32.

18. Ornoy A, Weinstein F, Ergaz Z (2015): Prenatal factors associated with autism spectrum disorder (ASD). Reprod Toxicol., 15 (56): 155-69

19. Zablotsky B, Anderson C, Law $P$ (2013): The association between child autism symptomatology, maternal quality of life, and risk for depression. Journal of Autism and Developmental Disorders, 43 (8): 19461955.

20. Volk H, Hertz-Picciotto I, Dewlike L et al. (2011): Residential proximity to freeways and autism in the CHARGE study. Environ Health Perspect., 119 (6): 873-7.

21. Modabbernia A, Velthorst E, Reichenberg A (2017): Environmental risk factors for autism: an evidencebased review of systematic reviews and meta-analyses. Molecular Autism, 8: 13.

22. Maimburg $\mathrm{R}$, Bech $\mathrm{B}$, Vaeth $\mathrm{M}$ et al. (2010): Neonatal jaundice, autism, and other disorders of psychological development. Pediatrics, 126 (5): 872-8.

23. Roumen $\mathbf{N}$ et al. (2009): Gastro intestinal symptoms in a sample of children with PDD autism. Dev Disord., 29 (2): 405-413.

24. Kheir N, Ghoneim O, Sandridge A et al. (2012): Quality of life of caregivers of children with autism in Qatar. Autism, 16 (3): 293-298.

25. Halladay A, Bishop S, Constantino J et al. (2015): Sex and gender differences in autism spectrum disorder summarizing evidence gaps and identifying emerging areas of priority. Molecular Autism, 6: 36-39.

26. Mandy W, Chilvers R, Chowdhury U et al. (2012): Sex differences in autism spectrum disorder: evidence from a large sample of children and adolescents. J Autism Dev Disord., 42 (1): 304-13.

27. Werling D, Geschwind D (2013): Sex differences in autism spectrum disorders. Current Opinion in Neurology, 26 (2): 146-153.

28. Lai D, Tseng Y, Hou Y et al. (2012): Gender and geographic differences in the prevalence of autism spectrum disorders in children: analysis of data from the national disability registry of Taiwan. Res Dev Disabil., 33 (3): 909-15.

29. Mayes S, Calhoun S (2011): Impact of IQ, age, SES, gender, and race on autistic symptoms. Res Autism Spectr Disord., 5 (2): 749-757.

30. Manohar H, Pravallika M, Kandasamy $P$ et al. (2018): Role of exclusive breastfeeding in conferring protection in children at-risk for autism spectrum 
https://ejhm.journals.ekb.eg/

disorder: results from a sibling case-control study. Journal of Neurosciences in Rural Practice, 9 (1): 132-138.

31. Previc F (2006): Prenatal influences on brain dopamine and their relevance to the rising incidence of autism. Med Hypotheses, 68 (1): 46-60.

32. Hvidtjørn D, Grove J, Schendel D et al. (2011): Risk of autism spectrum disorders in children born after assisted conception: a population-based follow-up study. J Epidemiol Community Health, 65 (6): $497-$ 502.

33. Kalkbrenner A, Braun J, Durkin M et al. (2012): Maternal smoking during pregnancy and the prevalence of autism spectrum disorders, using data from the autism and developmental disabilities monitoring network. Environmental Health Perspectives, 120 (7): 1042-1048 